\title{
Anemia hemolítica imunomediada em cães - relato de três casos
}

\section{Immune-mediated hemolytic anemia - report of three cases}

\author{
João Henrique Artero de Carvalho Leite'; \\ Luciana Curotto Nolasco de Carvalho ${ }^{2 *}$; Patricia Mendes Pereira ${ }^{3}$
}

\section{Resumo}

A anemia hemolítica imunomediada (AHIM) é um tipo comum de anemia em cães e gatos. A doença é mais comum em fêmeas caninas de meia-idade, especialmente Cocker Spaniel Americano. Os sinais clínicos estão associados com a anemia severa. Não há achados patognomônicos, mas a presença de anemia hemolítica em um cão jovem ou de meia idade, auto-aglutinação e esferócitos ou teste de Coombs positivo, eliminação de outros diagnósticos diferenciais e a resposta apropriada a terapia imunossupressora indicam AHIM. Apesar de inúmeras opções terapêuticas, os índices de mortalidade permanecem elevados. O objetivo do presente trabalho é relatar três casos graves de AHIM, ressaltando as modalidades terapêuticas e o prognóstico associado a elas.

Palavras-chave: Anemia hemolítica imunomediada, tratamento, prognóstico, cães

\begin{abstract}
Immune-mediated hemolytic anemia (IMHA) is a common type of anemia in dogs and cats. The disease é most common in middle-aged female dogs, especially American Cocker Spaniel. The clinical signs are associated with severe anemia. There is no pathognomonic test for IMHA, but the presence of hemolytic anemia in a young adult or middle age, autoagglutination and spherocytosis or positive results of Coombs test, elimination of any other underlying cause of anemia and an appropriate response to immunosuppressive therapy are suggestive of it. The aim of the present paper is to report of three cases of serious IMHA, and highlighting the therapeutic modalities and prognosis associated with them. Key words: Immune-mediate hemolytic anemia, treatment, prognosis, dogs
\end{abstract}

\footnotetext{
${ }^{1}$ Médico Veterinário Residente, setor de Clínica Médica de Animais de Companhia, Depto. Clínicas Veterinárias, Universidade Estadual de Londrina, UEL. E-mail: jhcarvalholeite@hotmail.com

${ }^{2}$ Médica Veterinária Residente, setor de Clínica Cirúrgica de Animais de Companhia, Depto. Clínicas Veterinárias, Universidade Estadual de Londrina, UEL. E-mail: lulucarv@hotmail.com

${ }^{3}$ Prof ${ }^{a}$. Adjunto do Departamento de Clínicas Veterinárias da Universidade Estadual de Londrina, Universidade Estadual de Londrina, UEL.E-mail: pmendes@uel.br

* Autor para correspondência
} 


\section{Introdução}

A anemia hemolítica imunomediada (AHIM) é uma anemia frequente na clínica de animais de companhia. A doença é causada pela destruição imunomediada dos eritrócitos e resulta em uma diminuição acentuada do volume globular (VG) (BALCH; MACKIN, 2007). A anemia hemolítica imunomediada pode ocorrer como um evento idiopático ou ser secundária a uma variedade de desordens infecciosas, neoplásicas entre outras (JAIN, 1986).

AHIM é mais comum em cães do que em gatos. Pode ocorrer em qualquer raça de cães, mas o Cocker Spaniel, Poodle, Sheepdog são os mais acometidos (MILLER; HOHENHAUS;
HALE, 2004). A raça Cocker Spaniel Americano representa aproximadamente um terço de todos os cães com AHIM (GIGER, 2000). A idade média de manifestação clínica é de seis anos, mas ela pode se desenvolver em animais de um a treze anos (WEINKLE; CENTER; RANDOLPH, 2005).

Os sinais clínicos frequentemente incluem fraqueza, intolerância ao exercício, apatia, anorexia, taquipnéia, dispnéia, vômito, diarréia e ocasionalmente poliúria e polidipsia. $\mathrm{O}$ exame físico revela mucosa pálida, taquipnéia, esplenomegalia, hepatomegalia, febre e linfoadenomegalia. Icterícia, hemoglobinúria e bilirrubinúria são comuns e facilmente observadas (REIMER; TROY; WARNICK, 1999). (Figura 1).

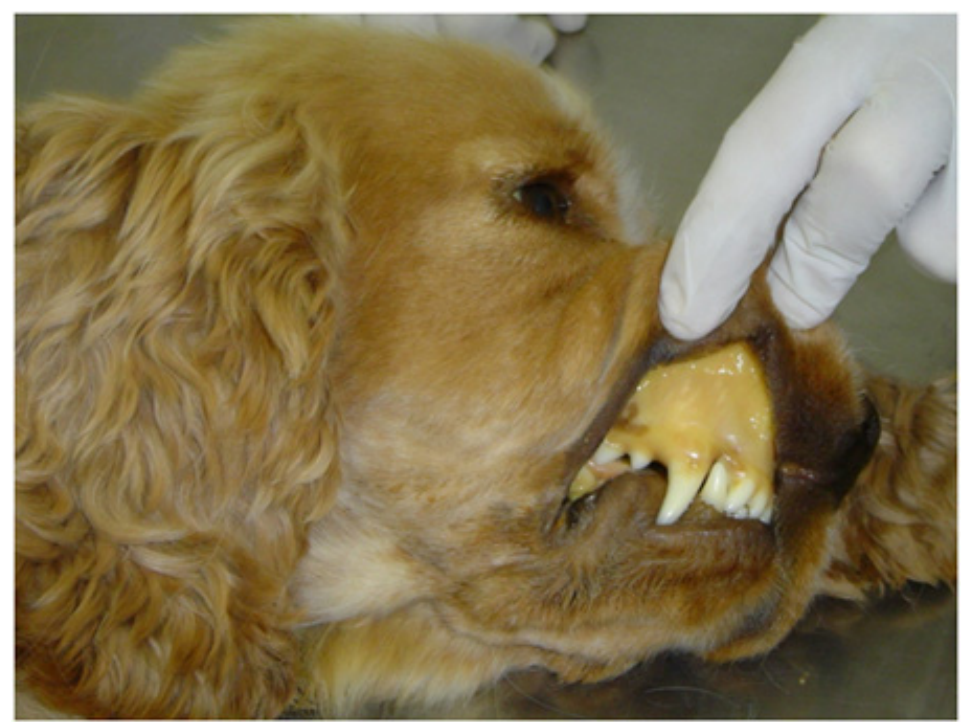

Figura 1. Mucosa oral pálida e ictérica de uma cadela Cocker Spaniel Americana com AHIM.

Distinguir entre AHIM primária e secundária é crucial para um tratamento efetivo. A doença primária requer terapia imunossupressora agressiva. A AHIM secundária raramente responde bem sem que a causa primária seja eliminada e em alguns casos, pode piorar com terapia imunossupressora (BURGESS et al., 2000).

Não há achados patognomônicos para a AHIM, mas sabe-se que anemia com VG inferior a $25 \%$, presença de hemoglobinúria e/ou bilirrubinúria, reticulocitose, auto-aglutinação, esferócitos, teste de Coombs positivo, além da eliminação de outras causas de base e uma resposta apropriada a terapia imunossupressora confirmam o diagnóstico de AHIM primária (SCOTT-MONCRIEFF et al., 2001). (Figura 2).

Uma vez diagnosticada, o tratamento suporte deve ser instituído. Muitos animais com destruição severa de hemácias inicialmente requerem hospitalização para monitorar e controlar a anemia. O objetivo do tratamento é estabilizar o VG inibindo a hemólise (CARR; PANCIERA; KIDD, 2002). 




Figura 2. Presença de auto-aglutinação em tubo de coleta sanguínea. Observa-se também o plasma intensamente amarelado indicando bilirrubinemia.

O tratamento suporte mais importante para pacientes com anemia severa é manter adequada a oxigenação tecidual. Esta deve ser realizada através da manutenção da volemia, já que alguns desses pacientes se encontram desidratados, além de repouso, oxigenoterapia e transfusão sanguínea. A transfusão sanguínea é indicada quando sinais clínicos como taquipnéia, dispnéia e taquicardia são intensos, demostrando severa hipóxia tecidual. Aproximadamente $70 \%$ a $90 \%$ dos pacientes com AHIM requerem transfusão sanguínea, com uma grande porcentagem recebendo múltiplas transfusões (BURGESS et al., 2000).

Idealmente, todos os animais que necessitam de transfusão sanguínea devem ser submetidos à reação cruzada. No entanto, a auto-aglutinação é comum em pacientes com AHIM e pode dificultar ou impossibilitar a acurácia do teste devido à incapacidade de diferenciar a auto-aglutinação de reação cruzada positiva (MELZER; WARDROP; HALE, 2003).

A despeito de controvérsias quanto ao fármaco ideal, é consenso que o tratamento da AHIM primária envolve o uso de agentes imunossupressores para reduzir a taxa de destruição eritrocitária mediada por anticorpos. Glicocorticóides como a prednisona e a dexametasona são os pilares da terapia da AHIM. A predinisona é tipicamente administrada nas doses de 1 a $2 \mathrm{mg} / \mathrm{kg}$ por via oral a cada 12 horas. A dexametasona pode ser utilizada também, particularmente nos animais que não podem receber medicação oral. Por ser sete a oito vezes mais potente que a prednisona, a dexametasona deve ser administrada em doses equipotentes (PLUMB, 2002).

A menos que efeitos colaterais sejam inaceitáveis, a dose do glicocorticóide não deve ser reduzida até que o VG do paciente esteja acima de $35 \%$ ou próximo aos valores de normalidade. Após a remissão, a dose deve ser mantida por uma a duas semanas e então reduzida de $25 \%$ a $50 \%$ a cada duas a quatro semanas. O tratamento poderá ser interrompido uma vez que a dose de prednisona for reduzida para 0,25 a $0,5 \mathrm{mg} / \mathrm{kg}$ a cada 48 horas (McCULLOUGH, 2003).

Doses imunossupressoras de glicocorticóides apresentam efeitos colaterais que podem limitar seu uso e frustrar proprietários. Efeitos colaterais como poliúria, polidipsia, polifagia e incontinência podem ser esperados e são especialmente evidentes 
em cães de grande porte. Complicações mais sérias incluem infecções secundárias, miopatia esteroidal e ulceração gástrica (PLUMB, 2002).

Terapia adicional com outros agentes imunossupressores deve ser administrada quando os glicocorticóides falham em induzir a remissão, causam efeitos colaterais inaceitáveis ou não podem controlar a doença mesmo quando administrados em dose elevada. Também devem ser incluídos no tratamento inicial em pacientes com autoaglutinação, hemólise intravascular e anemia não regenerativa, além de pacientes que necessitam de múltiplas transfusões (DAY et al., 2001).

A azatioprina apresenta excelentes resultados no tratamento da AHIM e pode melhorar o prognóstico dos pacientes que a recebem (KLAG; GIGER; SHOFER, 1993). A azatioprina é usada na dose de $2 \mathrm{mg} / \mathrm{Kg}$, por via oral a cada 24 horas. Por ter início de ação lento (sete a 14 dias), não deve ser utilizada isoladamente durante o tratamento inicial. Os efeitos colaterais são infrequentes e incluem anorexia, vômitos, diarréia, mielossupressão, hepatopatias e pancreatite (McCULLOUGH, 2003).

A ciclosporina é um imunomodulador que foi originalmente usado para prevenir o índice de rejeições em transplantes, mas agora é usado como agente imunossupressor para uma variedade de condições, incluindo fístula perineal, meningoencefalite granulomatosa e doença inflamatória intestinal (ADAMO; O' BRIEN, 2004). A dose imunossupressora recomendada para tratamento inicial é de $10 \mathrm{mg} / \mathrm{kg}$ a cada 12 a 24 horas (PLUMB, 2002), no entanto há poucos estudos que relatam a eficácia da ciclosporina em pequenos animais com AHIM (O'NEILL; EDWARDS; HOLLOWAY, 2004).

A ciclofosfamida é um agente alcalóide com potente efeito mielossupressor, que fez com que a droga fosse recomendada amplamente para $o$ tratamento de pacientes com AHIM severa. No entanto, ao contrário da azatioprina, não tem provado ser capaz de promover melhora clínica nos pacientes e está associada com redução das taxas de sobrevida (MASON et al., 2003). Os efeitos colaterais associados ao uso da ciclofosfamida são a anorexia, vômito, diarréia, mielossupressão e cistite hemorrágica. Por existirem outros agentes imunossupressores mais seguros e com melhor eficácia a ciclofosfamida não é mais recomendada para o tratamento da AHIM (ADAMO; O' BRIEN, 2004).

Sabe-se que a coagulação intravascular disseminada (CID) e o tromboembolismo, particularmente o pulmonar, é a complicação mais comum da AHIM (CARR; PANCIERA; KIDD, 2002). Aproximadamente $50 \%$ dos cães com AHIM estão em um quadro de hipercoagulabilidade por ocasião do diagnóstico (SCOTT-MONCRIEFF et al., 2001).

Muitos fatores que influenciam o desenvolvimento de trombos podem ocorrer em pacientes com AHIM. Lesão vascular pode desenvolver-se secundariamente a liberação de citocinas inflamatórias dos eritrócitos destruídos, bem como pela hipóxia tecidual (MCMANUS; CRAIG, 2001).

A coagulação intravascular disseminada é uma desordem trombohemorrágica sistêmica que tipicamente tem prognóstico ruim. $\mathrm{O}$ diagnóstico de CID é baseado em alterações hematológicas e hemostáticas como a diminuição do número de plaquetas, aumento do tempo de protrombina e tromboplastina parcial ativada, diminuição da concentração do fibrinogênio e aumento do número de esquisócitos (FELDMAN; MADEWELL; O’NEILL, 1999).

As atuais recomendações para prevenir a CID e o tromboembolismo incluem o uso da heparina não fracionada, heparina de baixo peso molecular ou doses baixas de aspirina. A heparina não fracionada pode ser administrada na dose de 250 $\mathrm{U} / \mathrm{kg}$ intravenoso ou subcutâneo a cada 6 horas. A dose é titulada através da monitoração do tempo da tromboplastina parcial (THOMPSON; SCOTT- 
MONCRIEFF; BROOKS, 2004). O uso da heparina de baixo peso molecular como a dalteparina e o enoxaparin está se popularizando na medicina humana, pois apresenta excelente eficácia com poucas complicações além de não necessitar de intensa monitoração do perfil hemostático (DUNN; CHARLAND; THORNELOE, 2004).

A administração de dose baixa de aspirina $(0,5 \mathrm{mg} / \mathrm{kg}$ a cada 24 horas) em combinação com prednisona e azatioprina foi relatada ser capaz de melhorar a sobrevida de cães com AHIM, mas não foi determinado se foi capaz de diminuir a prevalência da doença tromboembólica (WEINKLE; CENTER; RANDOLPH, 2005).

A taxa de mortalidade para a AHIM primária varia na literatura de $26 \%$ a $70 \%$ (KLAG; GIGER; SHOFER, 1993). A despeito desta variabilidade, há consenso que a doença tromboembólica é a maior causa de morte (MCMANUS; CRAIG, 2001). Weinkle, Center e Randolph (2005), observou que todos pacientes com AHIM necropsiados apresentaram tromboembolismo. As taxas de mortalidade irão cair dramaticamente quando métodos confiáveis para prevenir a doença tromboembólica forem estabelecidos (COHN, 2004).

Infelizmente o prognóstico para os cães com AHIM é reservado. A resposta completa ao tratamento pode levar semanas a meses, e alguns pacientes podem necessitar de tratamento contínuo ao longo da vida. A recidiva pode ocorrer a despeito de terapia prévia ou atual. Scott-Moncrieff et al. (2001), observou que 15\% dos cães com AHIM que sobreviveram por 60 dias apresentaram recidiva quando o tratamento foi descontinuado.

O presente artigo relata três cães com anemia hemolítica imunomediada em que todos pacientes vieram a óbito, demonstrando o caráter emergencial da enfermidade bem como seu baixo prognóstico.

\section{Relato dos Casos}

\section{Caso 1}

Um cão fêmea com 13 meses de idade, 31 quilos e mestiço Rottweiller. Apresentava prostração intensa, vômito e diarréia, desidratação moderada, icterícia e esplenomegalia com evolução de 48 horas. Quanto aos exames laboratoriais encontrou-se VG de 5,5\%, hiperglobulinemia, hipoalbuminemia, esferócitos e teste de Coombs positivo. Como tratamento optouse pela realização de transfusão sanguínea e a administração de dexametasona $0,3 \mathrm{mg} / \mathrm{kg}$ por via intravenosa. No entanto após 24 horas o animal veio a óbito.

\section{Caso 2}

Um cão fêmea com 5 anos de idade, 25 quilos, da raça Rottweiller. Apresentava prostração, vômito e diarréia, icterícia com evolução de duas semanas. Estava sendo tratada por um colega veterinário com doxiciclina sem obter melhora. Quanto aos exames laboratoriais encontrou-se VG de 11\%, reticulocitose, hemoglobinúria, bilirrubinúria e hipoalbuminemia. Como tratamento optouse por transfusão sanguínea e administração de dexametasona $0,2 \mathrm{mg} / \mathrm{kg}$ por via intravenosa. Após controle do vômito, iniciou-se administração de prednisona na dose de $2 \mathrm{mg} / \mathrm{kg}$ a cada 12 horas por via oral. Realizou-se monitoração diária do VG. Com nove dias de manutenção do tratamento e sem conseguir controlar a hemólise, VG de 13\%, associou-se azatioprina na dose de $2 \mathrm{mg} / \mathrm{kg}$, por via oral, a cada 24 horas. Após 48 horas da associação dos medicamentos o animal veio a óbito.

\section{Caso 3}

Um cão macho, com 5 anos de idade, sem raça definida. Apresentava prostração e hiporexia com evolução de 40 dias, sendo tratado com doxiciclina e diproprionato de imidocarb em 
colega veterinário sem obter melhora. Observouse icterícia, hepato-esplenomegalia, VG de $8 \%$, reticulocitose e hipoalbuminemia. Como tratamento realizou-se transfusão sanguínea e administração de prednisona $(2 \mathrm{mg} / \mathrm{kg}$ ) por via oral a cada 12 horas. O VG acompanhado diariamente, apresentou queda lenta e progressiva. Após a realização da terceira transfusão associou-se azatioprina, $2 \mathrm{mg} / \mathrm{kg}$ por via oral, a cada 24 horas. $\mathrm{O}$ animal apresentou piora clínica e ao exame radiográfico do tórax constatouse broncopneumonia. Diante do quadro clínico optou-se pela eutanásia.

\section{Discussão}

A taxa de mortalidade associada à anemia hemolítica imunomediada é alta, em torno de $50 \%$, a despeito do conhecimento sobre a enfermidade e o surgimento de novas drogas e regimes terapêuticos (MASON et al., 2003).

Estudos retrospectivos foram realizados recentemente para avaliar a eficácia terapêutica e índices de prognóstico. Reimer et al. (1999), em seu estudo retrospectivo com 70 cães com anemia hemolítica imunomediada relatou tempo de sobrevida de 974 dias em cães que receberam prednisona e azatioprina; 57 dias em cães que receberam somente prednisona; 28 dias em cães que receberam prednisona e ciclofosfamida e azatioprina.

Grundy e Barton (2001), avaliaram o tempo de sobrevida associado com vários tratamentos, incluindo prednisona, dexametasona, azatioprina, danazol, ciclosporina, ciclofosfamida, oxiglobulina e imunoglobulina intravenosa humana em 88 cães com anemia hemolítica imunomediada. Não foi observada diferença significativa nas taxas de mortalidade entre os animais que receberam um agente imunossupressor único ou associação destes, mas foi observado aumento do risco de morte com o uso de ciclofosfamida e oxiglobulina.

Em um recente estudo retrospectivo com 151 casos foi observado aumento na taxa de sobrevida em animais tratados com a combinação de glicocorticoídes, azatioprina e baixa dose de aspirina quando comparado com animais tratados apenas com glicocorticoíde e azatioprina (WEINKLE; CENTER; RANDOLPH, 2005).

Apesar da avaliação de grandes grupos de animais sob tratamento, a natureza retrospectiva destes estudos representa uma limitação significativa, pois não se observa critérios para a seleção dos grupos e pode-se predizer que animais severamente doentes tendem a receber maior combinação de fármacos, diminuindo, portanto, a taxa de sobrevida em protocolos de combinação de agentes imunossupressores (TOLL; ARONSOHN, 2003).

De forma geral, pode-se concluir que a utilização apenas de prednisona ou associação de prednisona com azatioprina (com ou sem dose baixa de aspirina) são as melhores opções para o tratamento inicial de muitos cães com anemia hemolítica imunomediada, além do que a ciclofosfamida não é indicada em muitos pacientes (MASON et al., 2003).

Com relação à conduta terapêutica adotada para os cães em relato pode-se observar a opção por iniciar o tratamento com a utilização da prednisona isolada com posterior associação de azatioprina, em média de cinco a sete dias após inicio do tratamento.

Com exceção do primeiro caso que apresentou evolução hiperaguda, os demais casos em relato apresentavam critérios para associação de azatioprina, pois os animais apresentavam auto-aglutinação, falha em induzir remissão com glicocorticoídes isoladamente e um animal necessitou de múltiplas transfusões.

Weinkle, Center e Randolph (2004), considerou o tratamento com azatioprina acessível. Tal fato não foi observado para os animais em relato, pois a restrição financeira dos proprietários foi um fator limitante para a adoção da droga ao tratamento, uma vez que o custo do medicamento, em apresentação comercial, foi proibitivo e há baixa disponibilidade do produto manipulado. 


\section{Considerações Finais}

A AHIM deve ser reconhecida como uma emergência médica. Em despeito das elevadas taxas de mortalidade e recidiva o diagnóstico precoce e a intervenção terapêutica adequada são fundamentais para o sucesso no tratamento.

\section{Referências}

ADAMO, F. P.; O' BRIEN, R. T. Use of cyclosporine to treat granulomatous meningoencephalitis in three dogs. JAVMA, Washington, DC, v. 225, n. 8, p. 1211-1216, 2004.

BALCH, A.; MACKIN, A. Canine Immune-Mediated Hemolytic Anemia: Pathophysiology, Clinical Signs, and Diagnosis. Compend. Contin. Educ. Pract Vet., Trenton, NS, v. 29, n. 4, p. 217-225, 2007.

BURGESS, K.; MOORE, A.; RAND, W.; COTTER, S. M. Treatment of immune-mediated hemolytic anemia in dogs with cyclosphosphamide. J. Vet. Intern. Méd., Philadelphia, v. 14, n. 4, p. 456-462, 2000.

CARR, A. P.; PANCIERA, D. L.; KIDD, L. Prognostic factors for mortality and thromboembolism in canine immune-mediated hemolytic anemia: A retrospective study of 72 cases. $J$ Vet Intern Med., Philadelphia, v. 16, n. 5, p. 504-509, 2002.

COHN, L. Immune mediated blood dyscrasias: therapeutic options. Proc Annu Forum Am Coll Vet Intern Med., v. 18, n. 3, p. 326-327, 2004.

DAY, T. K.; MACINTIRE, D. K.; MURTAUGH, R. J., MATHEUS, K. A. Differing opinions on treatment of immune-mediated hemolytic anemia. JAVMA, Washington, DC, v. 218, n. 9, p. 1414-1415, 2001.

DUNN, M.; CHARLAND, V.; THORNELOE, C. The use of a low molecular weight heparin in dogs (abstract). J Vet Intern Med., Philadelphia, v. 18, n. 3, p. 389, 2004.

FELDMAN, B. F.; MADEWELL, B. R.; O'NEILL, S. Disseminated intravascular coagulation: Antithrombin, plasminogen and coagulation abnormalities in 41 dogs. JAVMA, Washington, DC, v. 179, n. 2, p. 151-154, 1999.

GIGER, U. Regenerative anemias caused by blood loss or hemolysis, In: ETTINGER S. J.; FELDMAN, E. C. (Ed.). Textbook of veterinary internal medicine. 5. ed. St Louis: Editora Saunders, 2000. p. 1784-1804.
GRUNDY, S.; BARTON, C. Influence of drug treatment on survival of dogs with immune-mediated hemolytic anemia: 88 cases (1989-1999). JAVMA, Washington, DC, v. 218 , n. 4, p. 543-546, 2001.

JAIN, N. C. Hemolytic anemia of noninfectious origin. In: FELDMAN, B. F.; ZINKEL, J.G.; JAIN, N.C. (Ed.). Schalm's Veterinary Hematology. Philadelphia: Lea \& Febiger, 1986. p. 647-651.

KLAG, A.; GIGER, U.; SHOFER, F. Idiophatic immunemediated hemolytic anemia in dogs: 42 cases (19861990). JAVMA, Washington, DC, v. 202, n. 5, p. 783-788, 1993.

MASON, N.; DUVAL, D.; SHOFER, F. S.; GIGER, U. Cyclophosphamide exerts no beneficial effect over prednisone alone in the intial treatment of acute immunemediated hemolytic anemia in dogs: A randomized controlled clinical trial. $J$ Vet Intern Med., Philadelphia, v. 17 , n. 2, p. 206-212, 2MCCULLOUGH, S. Immunemediated hemolytic anemia. Understanding the nemesis. Vet Clin Small Anim., v. 33, n. 6, p. 1295-1315, 2003.

MCMANUS, P.; CRAIG, C. Correlation between leukocytosis an necropsy findings in dogs with immune mediated hemolytic anemia: 34 cases (1994-1999). JAVMA, Washington, DC, v. 218, n. 8, p. 1308-1313, 2001.

MELZER, K. J.; WARDROP, K. J.; HALE, A. S. A hemolitic transfusion reaction due to DEA 4 alloantibodies in a dog. J Vet Intern Med., Philadelphia, v. 17, n. 6, p. 931-933, 2003.

MILlER, S. A.; HOHENHAUS, A. E.; HALE, A. S. Case-control study of blood type, breed, sex, and bacteremia in dogs with immune-mediate hemolytic anemia. JAVMA, Washington, DC, v. 224, n. 2, p. 232235, 2004.

O'NEILL, T.; EDWARDS, G. A.; HOLLOWAY, S. Efficacy of combined cyclosporine A and ketoconazole treatment of anal furunculosis. J Small Anim Pract., v. 45, n. 5, p. 238-243, 2004.

PLUMB, D. Veterinary drug handbook. 4. ed. Ames: Iowa State University Press, 2002.

REIMER, M. E.; TROY, G. C.; WARNICK, L. D. Immune-mediated hemolytic anemia: 70 cases (19881996). JAAHA, Denver, CO, v. 35, n. 5, p. 384-390, 1999.

SCOTT-MONCRIEFF, J. C.; TREADWELL, N. G.; MCCULLOUGH, S. M.; BROOKS, M. B. Hemostatic abnormalities in dogs with primary immune-mediated hemolytic anemia. JAAHA, Denver, CO, v. 37, n. 3, p. 220-227, 2001. 
THOMPSON, M. F.; SCOTT-MONCRIEFF, J. C.; BROOKS, M. B. Effect of single plasma transfusion on thromboembolism in 13 dogs with primary immunemediated hemolytic anemia. JAAHA, Denver, CO, v. 40, n. 6, p. 446-454, 2004.

TOLL, J.; ARONSOHN, M. Prospective evaluation of medical therapy with or with-out splenectomy for the treatment of severe immune mediated hemolytic anemia (abstract). J Vet Inter Med., Philadelphia, v. 17, n. 3, p. $338,2003$.

WEINKLE, T. K.; CENTER, S. A.; RANDOLPH, J. F.; WARNER, K. L.; BARR, S. C.; ERB, H. N. Evaluation of prognostic factors, survival rates, and treatment protocols for immune-mediated hemolytic anemia in dogs: 151 cases (1993-2002). JAVMA, Washington, DC, v. 226, n. 11, p. 1869-1880, 2005. 